\title{
Exploring Approaches to Computer Cartography and Spatial Analysis in Cognitive Mapping Research: CMAP and MiniGASP Prototype Packages
}

\author{
Robert M. Kitchin \\ Department of Geography, University of Swansea Wales, UK, SA2 8PP.
}

\begin{abstract}
Much cognitive mapping data consists of spatial elements such as points, lines and polygons. This makes it possible to analyze such data with traditional spatial statistics and to visualise them using cartographic techniques. Using this approach can reveal 'hidden' spatial patterns and provide quantitative evidence which can be used in identifying, understanding and explaining cognitive mapping theories. This paper examines the role of computer cartography and spatial analysis in cognitive mapping research and the most appropriate approach to their implementation. The possible contribution of Geographical Information Systems (GISs) is investigated and the development and implementation of two stand-alone specialised packages, CMAP and MiniGASP, are outlined.
\end{abstract}

\section{INTRODUCTION}

Since the pioneering work of Lynch (1960), researchers from geography, cartography, psychology, planning and other social sciences have been interested in how we come to know and understand the spatial aspects of the geographic environment. Cognitive mapping research concerns the study of how we consciously, and more commonly subconsciously, acquire, learn, develop, think about and store data relating to our everyday geographic environment and representations of that environment (Downs and Stea, 1973; Kitchin, 1994a). Using controlled experiments, cognitive mapping researchers, seek to understand and explain the constituent components of spatial knowledge, how this knowledge is structured and stored, and the processes of learning and thought that people utilise. Methods of data collection and analysis vary from one subject to the next, but in geography there has traditionally been an analytical behavioural approach (Kitchin, 1996). This approach seeks to explain spatial behaviour by quantifiably determining the bases of cognition (knowledge and processes) that underlies action. The use of computer cartography, combined with spatial statistics, keeps within this tradition and extends the available tools of analysis and visualisation available, producing a wider range of possible results which can be interpreted and used in the investigation of cognitive mapping theory.

Cognitive mapping research has practical application in planning, policy making and education (Kitchin, 1994a). In relation to cartography, researchers are interested in how people understand maps and geographic material with the aim of finding ways to improve their skills in interpreting such information (e.g. Gerber and Kwan, 1994) and also to improve the maps and material so that they are easier to understand (Lloyd and Steinke, 1986). Psychologists (e.g. Thorndyke and Statz, 1980) and geographers (e.g. Gilmartin and Patton, 1984) have both investigated ways to improve map reading and interpretation skills and Thorndyke (1981) and MacEachren (1991) have examined how to redesign maps to increase their usability. Similar arguments have been advanced for improving the databases and output from Geographic Information Systems (Mark and Gould, 1991; Medyckyj-Scott and Blades, 1992). These issues have particular relevance to geographic education for children (see Matthews, 1992), and mobility training and technical aid design (e.g. tactile maps and talking signs) for people with visual impairments or blindness (see Golledge et al., 1991; Ungar et al., 1995).

\section{WHAT DO COMPUTER CARTOGRAPHY AND SPATIAL STATISTICS OFFER COGNITIVE MAPPING RESEARCH?}

Combining computer cartography with spatial statistics gives an opportunity to study the 'hidden' nature of cognitive mapping data and to provide precise, quantitative evidence which can be used in identifying, comprehending and explaining cognitive mapping theories. Spatial products, the externalised form of cognitive map knowledge, have many of the properties of traditional paper maps as they consist of spatial features such as points, lines and polygons (e.g. a sketch map). They can, therefore, be converted to a digitised form suitable for display using 
computer cartography and analysis using spatial statistics. Computer cartography permits traditional paper-based cartography to be performed on a computer where sophisticated software, as found in many GISs, allow easy manipulation and storage. Spatial statistics allow the detection and description of spatial patterns and properties. As such, they can provide information which allows the modelling, and further understanding, of the spatial processes underlying a subject area. In particular, they can provide precise evidence concerning the geometry and distortions of spatial products. Such information can help us understand how we think about space and provide results which can be used in the many applications detailed above.

Until recently, computing power has restricted the use of both computer cartography and spatial analyses in cognitive mapping research, although cartographic techniques, such as the plotting of standard deviation ellipses (see Buttenfield, 1986; Golledge et al., 1987), and spatial analyses, such as bidimensional regression (see Tobler, 1976; Kitchin, 1993; Murphy, 1978), are well developed. However, the lack of computing power has restricted analyses to aggregate data sets due to the time consuming nature of computing individual results. This practice has restricted the utility of the analyses and a recent investigation (Kitchin and Fotheringham forthcoming) has found that the strategy of aggregation not only leads to alternative results, but also masks high variability in cognitive map knowledge at the individual level. Recent developments in personal computing now means that many data sets can be analyzed, in a variety of ways and in a short period of time. At present, cognitive mapping researchers seeking computer assisted analysis have two available options: generic GISs or specialised software. Both these options are explored.

\section{THE GIS APPROACH}

GISs initially seem ideally suited to cognitive mapping research because they are packages designed to store, manipulate and analyze, spatial data. GISs and cognitive mapping thus appear to have much in common, with GISs being computer systems specifically designed to process data concerning the everyday geographic environment, and cognitive mapping concerning how we think, store, produce and utilise such data. Both cognitive map knowledge and GIS's contain data concerning spatial relationships and attributive data, assigned to specific places which exist within a space-time framework.

There has, however, been very little actual research concerning how GISs could aid the study of cognitive mapping and most of what has been written is concerned with possible applications rather than actual practical studies. What practical work that exists consists of one specific study by Dramowicz (1990) and the behavioural geography work of Aitken and colleagues (Aitken and Prosser, 1990; Aitken et al., 1990; Aitken et al., 1993). This section reviews this work and outlines the potential uses and disadvantages of applying GIS to cognitive mapping research.

\section{How could a raster GIS be of use to cognitive mapping?}

Aitken and colleagues (Aitken and Prosser, 1990; Aitken et al., 1990; Aitken et al., 1993) have studied the residential cognition of neighbourhood and form, concentrating on measures of spatial familiarity and experience. They used ARC/INFO, a vector GIS to display their data, but their analysis is more easily discussed within a raster context because of its grid-based nature. They collected their data by asking respondents to state how familiar, or experienced, they were with each cell of a transparent grid overlaid upon an aerial photograph. They then structured this data into a GIS and analysed it using a moving window spatial autocorrelation statistic to describe the underlying structure of the data. This was made possible by initially organising the data into a grid-based form at the data collection stage. This is an example of a behavioural-based GIS database being implemented and used in a planning study. The problem with such an approach lies in the fact that cognitive mapping data (e.g. sketch map) is often vector in format and it would be difficult to rasterise such data. However, once in a raster format other raster GIS functions may be of use. These could include the many classification methods, especially those that involve some sort of measure of uncertainty or 'fuzziness' (e.g. evidential classifiers), which is an inherent part of behavioural data.

\section{How could a vector GIS be of use to cognitive mapping?}

Dramowicz (1990) used a vector GIS to investigate the nature of cognitive maps. He collected a series of hand drawn data at the varying scales which were later digitised for analysis within a GIS. Unfortunately Dramowicz only used the GIS as a storage and visualisation device. Dramowicz visually interpreted the spatial products by using manipulations such as reclassification to alter displays, rather than trying to perform any complex analysis of individual maps. The main advantage of such an approach being time-saving on simple operations such as overlay and reclassification.

Extending beyond Dramowicz's practical work, cognitive mapping and GIS have generally been discussed purely at a theoretical level. Golledge (1992) has discussed the possibility of using GIS in conjunction with a route scheduler to collect data for wayfinding studies. The results could then be displayed and compared with data determined by a network based model for use in planning. The results could also be modelled using a host of other cognitive variables believed to influence route decision making, such as the socio-economic characteristics of a neighbourhood, crime levels and familiarity. It should thus be possible to determine why people choose certain routes. The problem with this GIS approach is that very little analysis can actually be done beyond overlay, and any analysis of the geometry and distortions of the respondent's cognitive map are impossible. GISs are currently not well suited to the analysis of cognitive map data beyond display and visual analysis because they lack any suitable spatial analysis techniques.

\section{THE ANALYTICAL COMPUTER CARTOGRAPHY APPROACH: CMAP AND MiniGASP}

As an alternative to GIS, the analytical computer cartography approach of Gale (1982) seems an appropriate and a useful approach to cognitive mapping. As suggested 
above, GISs are capable of displaying and manipulating cognitive map data but they cannot perform sophisticated analyses of these data, nor do they offer specialised data collection methods. Software is therefore needed that can combine the computer cartography visualisation and manipulation aspects of GISs, and the analytical capabilities of spatial statistics. There are five ways such visualisation and analysis can be combined (see Table 1).

At present, the full integration of spatial statistics into GISs is unlikely. Market demand is low beyond academia and the development and implementation costs are relatively high for limited potential profits. Loose and close coupling have the advantages of providing highly sophisticated mapping and database facilities with specialised-analytical software (Rowlingson and Diggle, 1991) and this avenue is currently being explored by many academics (see Gatrell et al., 1994). The addition of spatial statistics into an established statistical package such as MINITAB by using specialised macros is only suitable for teaching purposes and often suffers from poor graphics and little mapping capabilities.

The approach which seems to have most utility to cognitive mapping research is the stand-alone specialised package/program. This approach has advantages because the system can be customised to the user's needs, providing the functionality required and other specialised features relating to data collection and analysis. Over a period of three years, two packages, Cognitive Mapping Analysis Package (CMAPl) (Kitchin, 1994b) and Geographical Analysis Statistics Package (MiniGASPI) (Bratt and Kitchin, 1994) were developed. Both packages are capable of the sophisticated analysis and the specialised visualisation needed in analytical cognitive mapping research. Both packages provide a range of data collection, analysis, manipulation and visualisation functionality.

CMAP is a refined and updated version of SCAMS (Kitchin, 1992) a command-line driven program developed as a specialised package to collect, display and analyze cognitive mapping data. Using SCAMS, data could be collected from respondents in four main ways: collected on paper and digitised using another package; typed into a file as a series of coordinates or as distance and direction units; digitised onto the screen with points representing places overlain upon a base map; or digitised onto ratio scaling lines and directional compasses. Digitising straight to the screen has a number of advantages such as speed of collection and the ability to easily correct errors. Familiarity

Table 1. Methods of achieving computerised spatial data analysis.

1. Loose-coupling - spatial statistics software is made compatible with GIS software data which is exported for analysis e.g. 'SPLANCS' (Rowlingson and Diggle, 1993).

2. Close-coupling - calling a spatial analysis routine from within the GIS (see Gatrell et al., 1994).

3. Full integration of spatial statistics into a GIS.

4. Specialised stand-alone packages such as the 'Geographical Analysis Machine' (Openshaw et al.,1987) and 'SpaceStat' (Anselin, 1991).

5. The addition of spatial statistics into established statistics packages such as MINITAB through specialised macros (see Griffith, 1992). data could also be collected and used in the analysis. Users could convert their data from coordinates to distance and direction estimates and vice versa, and to aggregate groups of data together. Analysis could then be performed using bidimensional regression upon coordinate data (see Tobler, 1976; Kitchin, 1993), and linear regression upon distance and direction data, and the results could be displayed graphically. A full help system accompanied the package to guide the user. The system was 'idiot-proofed' to a basic standard, allowing the use of suitable files and possible commands, e.g. it was impossible to place a city in the sea. Although able to process any cognitive mapping data, as long as it was in either a distance and direction or coordinate format, it had limited flexibility because it was originally designed to process British scale data and as such the working area was rectangular with a fixed coordinate area.

CMAP offers no data collection facilities but a wider range of analysis, manipulation and data types. For example, data collected from all the exercises displayed in Figure 1 can be processed, displayed graphically and spatially analysed. The program has three principle advantages. Firstly, analyses can be carried out at the individual level, with macros allowing hundreds of data sets to be analysed in a couple of minutes, and at an aggregate level. Secondly, using a simple database structure CMAP can compensate for missing data and also allow defined data, such as guesses and those below a certain familiarity threshold, to be excluded from the analysis. Thirdly, using specialised macros the results from the individual analyses can be combined into ASCII files for analysis using conventional parametric tests in a standard package such as MINITAB.

The program is controlled through the use of both command-lines and the mouse. Analysis includes bidimensional regression, Waterman and Gordan's (1984) distortion calculations, standard deviation ellipses upon aggregated data (see Buttenfield, 1986; Golledge et al., 1987), and new analysis techniques for close procedure and recognition (orientation specification) data consisting of individual and aggregate accuracy scores (see Kitchin, forthcoming). Manipulation routines allow data to be congruently displayed through scale standardisation, rotation and translation; to aggregate all the different data types including those data sets of varying lengths and contents; reflect MDS data which has been reversed and the standardisation of lines to allow comparison. Projective convergence (or resection) data analysis (see Kirasic et al., 1984; Kitchin, forthcoming) has also been included and this uses distance and directional estimates to work out the coordinates of locations.

MiniGASP is an interactive DOS-based 'windows' CAL (computer-assisted learning) package aimed at teaching spatial statistics and concepts to undergraduates. It also represents a continuation of the work first started in SCAMS. Although not dedicated to cognitive mapping research it allows the calculation of spatial statistics not included in CMAP. MiniGASP allows the collection of point, line and polygon data either through on-screen digitising or as edited data files. This means that analysis of cognitive mapping data can progress beyond traditional point-based analysis to include line and polygon inquiry. For example, the description of properties such as length, 
(a)

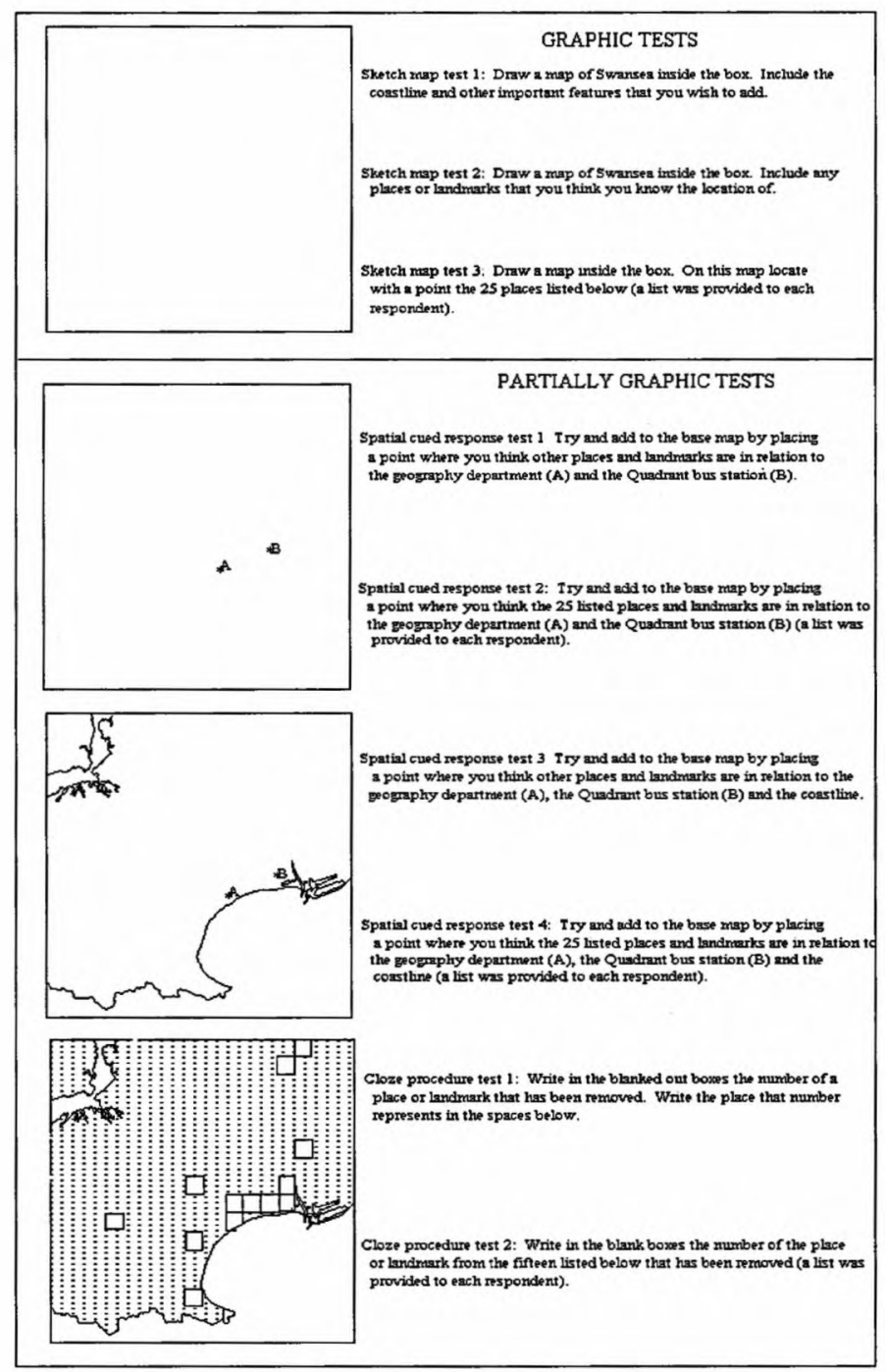

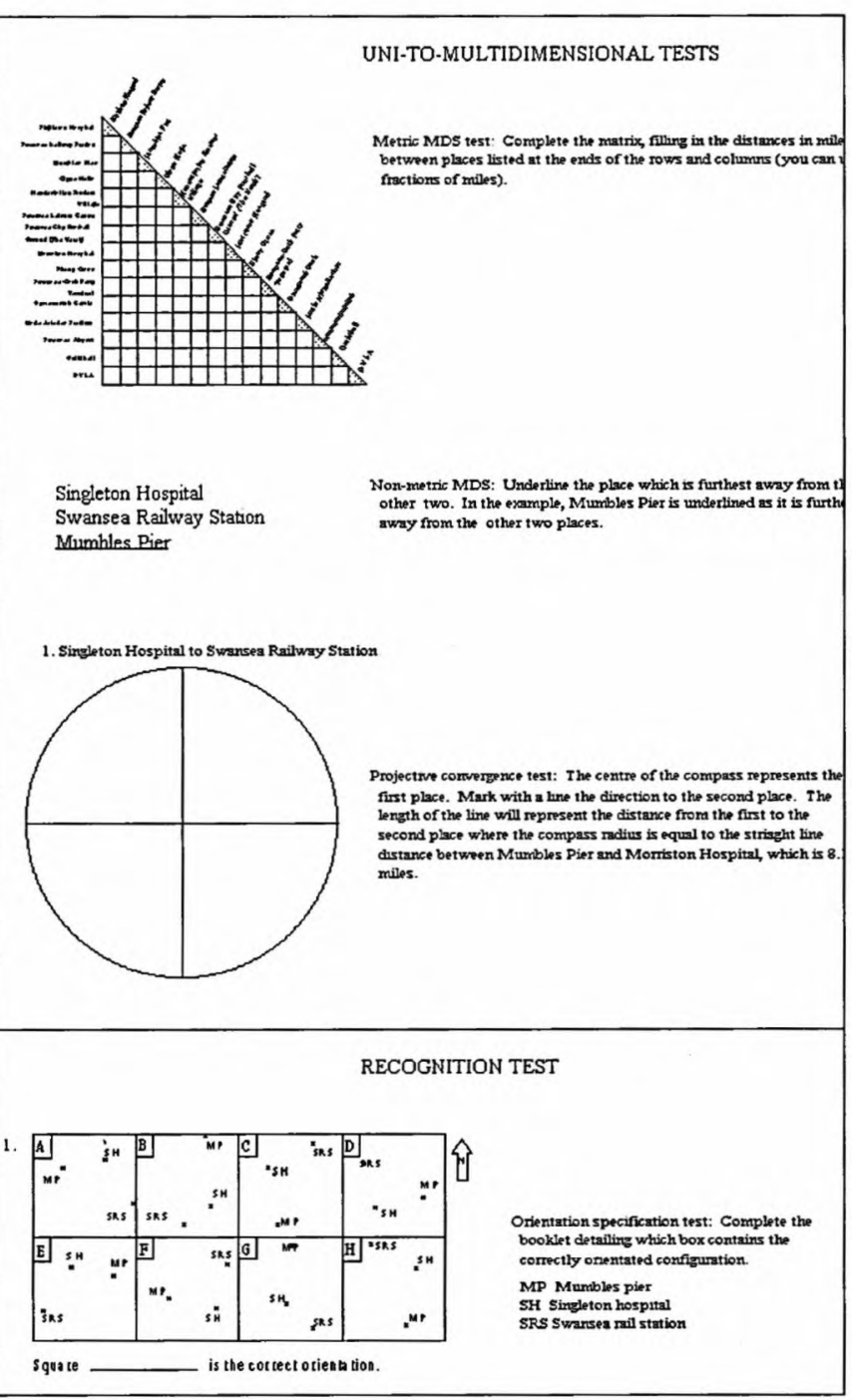

(b)

Figure 1. (a) The graphic and partially graphic tests CMAP can display and analyze; (b) The uni-to-multidimensional and orientation specification tests CMAP can display and analyze

sinuosity, area and shape can be calculated. These have rarely been included in analysis of cognitive mapping data because of the difficulty and time-consuming nature of hand calculation. MiniGASP also has the capability to display remote sensing or aerial photographs and this allows respondents to identify named locations. It is hoped that the analytical capabilities of this package may lead to new discoveries in cognitive mapping research. MiniGASP is currently a working prototype but will eventually perform a host of comparative and descriptive statistics.

\section{CONCLUSIONS}

This paper has aimed to explore the possible contribution of computer cartography and spatial statistics to cognitive mapping research and the most appropriate approach to achieve successful implementation. Using the computer as a visualisation and analytical tool in the analysis of cognitive mapping has three main advantages. Firstly, it provides precise, quantitative evidence regarding the geometry and distortions of spatial products. Secondly, computers are efficient stores'of data and allow great time savings on data analysis, that if calculated by hand would be difficult and tedious to compute. Thirdly, data can be visualised from a new perspective and manipulated easily. When combined, these advantages suggest that computer cartography and spatial analyses have the potential to create a greater understanding of cognitive map knowledge for a variety of real world applications. GISs at present are unsuitable for this purpose, lacking the sophisticated spatial analysis which is needed. A more suitable alternative, is the development of specialised packages, capable of the collection, analysis and visualisation of cognized data. CMAP and MiniGASP are prototype packages that adopt this specialist approach. Both have been successfully used, in a large study of student's cognitive map knowledge, to process and analyse data from 312 respondents, 170 of which completed 4 of the tests displayed in Figure 1 (Kitchin, 1995; Kitchin, forthcoming).

\section{NOTES}

${ }^{1}$ CMAP and MiniGASP, a demonstration version of GASP have been released as academic shareware and are available at no cost from the author with detailed manuals (CMAP, pp. 38; MiniGASP, pp. 22). Both packages were programmed using Turbo Pascal.

\section{REFERENCES}

Aitken, S.C., and Prosser, R (1990). 'Residents spatial knowledge of neighbourhood continuity and form', Geographical Analysis, 22, 4, 301-325. 
Aitken, S.C., Chandler, R., Prosser, R. and Stutz, F.P. (1990). 'Investigating the structure of neighbourhood cognition through familiarity and experiential surfaces', Working Paper presented to the IGU commission on Mathematical Models, Boston, Massachusetts.

Aitken, S.C., Stutz, F., Prosser, R., and Chandler, R. (1993). 'Neighbourhood integrity and residents familiarity: Using a Geographic Information System to investigate place identity', Tijdschrift Voor Econmische en Sociale Geografie, 1-12.

Anselin, L. (1991). 'SpaceStat: a program for the analysis of spatial data', NCGIA, Department of Geography, University of California Santa Barbara, USA.

Bratt, M., and Kitchin, R.M. (1994). 'MiniGASP', Public domain shareware. University of Wales Swansea.

Buttenfield, B.P. (1986). 'Comparing distortion on sketch maps and MDS configurations', Professional Geographer, 38, 3, 238-246.

Downs, R.M., and Stea, D. (1973). 'Theory', in Image and Environment (Eds. R.M. Downs and D. Stea), pp. 8-24, Aldine. Chicago.

Dramowicz, K. (1990). 'Application of GIS for the cognitive mapping: GIS for the 1990s', Proceedings of the CISM.

Gale, N. (1982). 'Some applications of computer cartography to the study of cognitive configurations', Professional Geographer, 34, 3, 313-321.

Gatrell, A.C., Openshaw, S., Brunsdon, C., Charlton, M., Rowlingson, B., and Rao, L. (1994). 'A spatial analysis toolkit for ARC/INFO', Paper presented at the IBG Annual Conference, University of Nottingham.

Gerber, R., and Kwan, T. (1994). 'A phenomenological approach to the study of pre-adolescents' use of maps in a wayfinding exercise in a suburban environment', Journal of Environmental Psychology, $14,265-280$.

Gilmartin, P., and Patton, J.C. (1984). 'Comparing the sexes on spatial abilities: map-use skills', Annals of the Association of American Geographers, 74, 605-619.

Golledge, R.G. (1992). 'Place recognition and wayfinding: making sense of space', Geoforum, 23, 2, 199-214

Golledge, R.G., Loomis, J.M., Klatzky, R.L., Flury, A., and Yang, X-L. (1991). 'Designing a personal guidance system to aid navigation without sight: Progress on the GIS component', International Journal of Geographical Information Systems, 5, 373-396.

Golledge, R.G., Gale, N. and Richardson, G.D. (1987). 'Cognitive maps of cities II: Studies of selected populations', National Geographical Journal of India, 33, 1-16.

Griffith, D.A., (1992). 'Teaching spatial statistics to geographers using MINITAB', Journal of Geography in Higher Education, 16, 1, 45-59.

Kirasic, K.C., Allen, G., and Siegel, A.W. (1984). 'Expression of configurational knowledge of large-scale environments: student's performance of cognitive tasks', Environment and Behaviour, 16, 687-712.

Kitchin, R.M. (1992). 'The Application of GIS to Spatial Cognitive Studies: SCAMS - A Preliminary System: A study to investigate methods of data collection and analysis', Unpublished MSc Thesis.
Kitchin, R.M. (1993). 'Using bidimensional regression to analyze cognitive maps', Swansea Geographer, 30, 33-50.

Kitchin, R.M. (1994a). 'Cognitive maps: what are they and why study them?', Journal of Environmental Psychology, 14, 1-19.

Kitchin, R.M. (1994b). CMAP (Cognitive Mapping Analysis Package). Public domain shareware. University of Wales, Swansea.

Kitchin, R.M. (1995). 'Issues of Validity and Integrity in Cognitive Mapping Research: Investigating Configurational Knowledge', Unpublished $\mathrm{PhD}$ Thesis, University of Wales, Swansea.

Kitchin, R.M. (1996). 'Increasing the integrity of cognitive mapping research: appraising conceptual schemata of environment-behaviour interaction', Progress in Human Geography, 20, 56-84.

Kitchin, R.M., forthcoming. 'Methodological convergence in cognitive mapping research: investigating configurational knowledge', Journal of Environmental Psychology.

Kitchin, R.M., and Fotheringham, A.S. forthcoming. 'Aggregation issues in cognitive mapping research', Professional Geographer.

Lloyd, R.E., and Steinke, T.R. (1986). 'The identification of regional boundaries on cognitive maps', The Professional Geographer, 38, $2,149-159$.

Lynch, K. (1960). The Image of the City, MIT Press, Cambridge.

MacEachren, A.M. (1991). "The role of maps in spatial knowledge acquisition', The Cartographic Journal, 28, 152-162.

Mark, D.M., and Gould, M.D. (1991). 'Interacting with Geographic Information: A commentary', Photogrammetric Engineering and Remote Sensing, 57, 1427-1430.

Matthews, M.H. (1992). Making Sense of Place: Children's Understanding of Large-Scale Environments, Harvester Wheatsheaf, Hemel Hempstead.

Medyckyj-Scott, D., and Blades, M. (1992). 'Human spatial cognition: Its relevance to the design and use of spatial information systems', Geoforum, 23, 215-226.

Murphy, J. (1978). 'Measures of map accuracy assessment and some early Ulster maps', Irish Geography, 11, 89-101

Openshaw, S., Charlton, M., Wymer, C., and Craft, A. (1987). 'A mark 1 geographical analysis machine for the automated analysis of point data sets', International Journal of Geographical Information Systems, 1, 335-358.

Rowlingson, B.S., and Diggle, P.J. (1993). 'SPLANCS: spatial point analysis in S-PLUS', Computers and Geosciences, 19, 627-655

Thorndyke, P.W. (1981). 'Distance estimation from cognitive maps', Cognitive Psychology, 13, 526-550.

Thorndyke, P.W., and Statz, C. (1980). 'Individual differences in procedures for knowledge acquisition from maps and navigation' Cognitive Psychology, 12, 137-175.

Tobler, W.R. (1976). 'The geometry of mental maps', in Spatial Choice and Spatial Behaviour (Eds. R.G. Golledge and G. Rushton), pp. 69-82, Ohio State University Press, Columbus Ohio.

Ungar, S., Blades, M., and Spencer, C. (1995). 'Visually impaired children's strategies for memorizing a map', British Journal of Visual Impairment, 13, 27-32.

Waterman, S., and Gordon, D. (1984). 'A quantitative-comparative approach to analysis of distortion in mental maps', Professional Geographer, 36, 3, 326-337. 